\title{
SALMONELLA DANS LA FILIÈRE PORCINE À LA RÉUNION : ÉTUDE LONGITUDINALE DE LA FERME À LA FOURCHETTE
}

\author{
Salmonella in PIG PROduction, ReUniOn IsLAND: \\ LONGITUDINAL STUDY FROM FARM TO FORK
}

\section{Salmonella en la PRODUCCIÓN PORCINA, ISLA DE LA ReUnión: ESTUDIO LONGITUDINAL DE LA GRANJA AL TENEDOR}

\author{
C. Tessier ${ }^{1,2,3,4,5 *}$ L. Atiana ${ }^{2,3,4}$ E. Cardinale ${ }^{2,3,4}$ M. Denis 6,7
}

\author{
Mots-clés : Viande porcine - Salmonella - Infection - \\ Contamination - Réunion. \\ Keywords: Pork - Salmonella - Infection - \\ Contamination - Reunion. \\ Palabras clave: Carne de cerdo - Salmonella - Infección - \\ Contaminación - Reunión.
}

\begin{abstract}
Salmonella est le principal agent zoonotique pathogène en Europe après Campylobacter (2). Les produits à base de viande de porcs sont souvent incriminés. Cependant, ce pathogène est difficilement détectable en élevage puisque le portage est asymptomatique. Malgré une localisation tropicale, la filière porcine de la Réunion est également concernée par ce problème (1).
\end{abstract}

L'objectif de cette étude a été d'identifier les différentes voies de contamination tout au long de la filière porcine. Pour cela, un lot de porcs (sélection de trois truies par lot et cinq porcelets par truie) dans trois élevages positifs en salmonelles ont été suivis depuis la mise bas jusqu'au produit fini. Au stade de l'élevage, l'excrétion de salmonelles a été mise en évidence par des prélèvements individuels de fèces quelques jours après la mise bas, puis pendant la nurserie, le postsevrage et l'engraissement. Des échantillons d'aliment et d'eau ainsi que des prélèvements d'environnement par chiffonnage (salles et couloir avant transfert, abords de l'élevage, autres productions animales sur le site) ont également été collectés à chaque visite.

Au stade de l'abattoir et de l'atelier de découpe, des chiffonnettes ont été prélevées à différentes étapes avant le passage du lot (camion de transport et box d'attente après nettoyage et désinfection, matériel sur la chaîne d'abattage et de découpe avant le

1. Sicabat, 3 avenue Charles Isautier, 97410 Saint-Pierre, Réunion, France. 2. Cirad, UMR Cmaee, 2 rue Maxime Rivière, 97491 Sainte-Clotilde, Réunion, France.

3. INRA, UMR Cmaee, 73 rue Jean-François Breton, 34398 Montpellier.

4. Centre de recherche et de veille sur les maladies émergentes dans l'océan Indien, Cyroi, 97491 Sainte-Clotilde Cedex, Réunion, France.

5. Université de la Réunion, 97715 Saint-Denis, Réunion, France.

6. Anses,Unité HQPAP, BP 53, 22440 Ploufragan, France.

7. Université de Bretagne occidentale, Brest, France.

* Auteur pour la correspondance

E-mail : claire_tessier@outlook.fr début de l'abattage du jour) ainsi que sur les animaux identifiés (dos des porcs à l'entrée à l'abattoir et carcasses après la deuxième flagelleuse, avant et après le froid-choc). Les caecums ainsi que des produits de découpes (côte et rouelle de porc) provenant du lot d'étude ont également été échantillonnés.

La méthode de détection de Salmonella a été adaptée à partir de la norme ISO 6579 Annexe D (4). La quantification de Salmonella par la méthode mini-MSRV (3) a également été réalisée sur les prélèvements de fèces collectées pendant la croissance à l'élevage. Tous les isolats ont été sérotypés et génotypés par électrophorèse sur gel en champ pulsé (PFGE) en utilisant l'enzyme Xbal (5).

Sur les 879 échantillons collectés, 27 p. 100 (79/293), 28,2 p. $100(82 / 291)$ et 22,7 p. $100(67 / 295)$ étaient positifs respectivement pour les élevages A, B et C. Les 908 isolats appartenaient à 55 pulsotypes et 14 sérotypes ; S. 4,[5],12:i:-, $S$. Rissen, $S$. Typhimurium et $S$. Livingstone ont été prédominants (tableau I). Les tableaux II et III montrent la distribution des pulsotypes de l'élevage B. Au stade de la maternité, tous

\section{Tableau I}

Distribution des isolats et des pulsotypes de Salmonella détectés pendant le suivi d'élevages de porcs à l'abattoir à la Réunion

\begin{tabular}{lrc} 
Sérotypes de Salmonella & Nb. d'isolats & Nb. de pulsotypes \\
\hline 4,[5],12:i:- & 312 & 23 \\
Rissen & 231 & 4 \\
Typhimurium & 105 & 7 \\
Livingstone & 99 & 7 \\
Weltevreden & 58 & 4 \\
Derby & 35 & 1 \\
London & 34 & 1 \\
Senftenberg & 12 & 2 \\
Newport & 7 & 1 \\
Bredeney & 4 & 1 \\
Blockley & 4 & 1 \\
Give & 3 & 1 \\
Corvallis & 2 & 1 \\
Durban & 2 & 1
\end{tabular}


les porcelets étaient négatifs et seulement une truie a excrété Salmonella à une seule occasion. L'infection des porcs a été observée après le sevrage (élevages $A$ et $B$ ) mais aussi pendant I'engraissement (élevage C). L'infection à Salmonella a duré entre 34 et 40 jours en moyenne, avec des taux d'excrétion très variables pendant la croissance des animaux. L'excrétion a été plus élevée dans les caecums que dans les fèces. En élevage, les pulsotypes excrétés par les porcs ont été identiques à ceux isolés des box après le nettoyage et la désinfection, et du couloir avant le transfert des animaux.

\section{Tableau II}

Distribution des pulsotypes de Salmonella détectés pendant le suivi de l'élevage de porcs B (maternité, postsevrage et engraissement) à la Réunion

\begin{tabular}{|c|c|c|c|c|c|c|c|c|c|c|c|c|}
\hline & & $\mathrm{Ma}$ & ernité & & & stsevra & & & & raissem & & \\
\hline & $\begin{array}{l}\text { Ap } \\
\text { ND }\end{array}$ & Ent & $\begin{array}{c}\mathrm{MB}+4 \\
\text { jours }\end{array}$ & Sev & $\begin{array}{l}\text { Ap } \\
\text { ND }\end{array}$ & $\begin{array}{c}+2 \\
\text { sem. }\end{array}$ & Sor & $\begin{array}{l}\text { Ap } \\
\text { ND }\end{array}$ & $\begin{array}{c}+1 \\
\text { mois }\end{array}$ & $\begin{array}{c}+1 \\
\text { mois }\end{array}$ & $\begin{array}{c}+1 \\
\text { mois }\end{array}$ & Sor \\
\hline Porcs & & - & - & - & & M5 & M5 & & $\begin{array}{c}\text { M5 } \\
\text { M11 }\end{array}$ & $\begin{array}{l}\text { Li5 } \\
\text { Li6 } \\
\text { M5 }\end{array}$ & M17 & M5 \\
\hline Salle & - & - & - & $\mathrm{T} 1$ & M5 & M5 & M5 & M21 & $\begin{array}{l}\text { M3 } \\
\text { M4 } \\
\text { M5 }\end{array}$ & $\begin{array}{l}\text { Li5 } \\
\text { M4 }\end{array}$ & $\begin{array}{l}\text { M11 } \\
\text { M17 }\end{array}$ & M5 \\
\hline Couloir & $\mathrm{T} 4$ & & & & $\begin{array}{c}\text { M5 } \\
\text { T2 }\end{array}$ & & & - & & & & $\begin{array}{l}\text { M5 } \\
\text { M9 }\end{array}$ \\
\hline Quai embarquement & & & & & & & & & & & & $\begin{array}{c}\text { M3 } \\
\text { M22 } \\
\text { M23 }\end{array}$ \\
\hline Abords & & & Li5 & $\mathrm{T} 1$ & & Li5 & & & M3 & T3 & - & \\
\hline Autres productions & & & $\begin{array}{l}\text { M9 } \\
\text { M10 }\end{array}$ & - & & & - & & $\begin{array}{l}\text { Du1 } \\
\text { M3 } \\
\text { M5 }\end{array}$ & $\begin{array}{l}\text { M5 } \\
\text { M6 }\end{array}$ & - & M5 \\
\hline
\end{tabular}

Ap ND : après nettoyage et désinfection ; Ent : entrée ; MB : mise bas ; Sev : sevrage ; Sor : sortie

Les différents sérotypes de Salmonella ont été indiqués par des lettres (Li : Livingstone ; M : 4,[5],12:i:- ; T : Typhimuirum ; Du : Durban) suivies du numéro du pulsotype.

- : Pas de Salmonella

\section{Tableau III}

Distribution des pulsotypes de Salmonella détectés pendant le suivi de l'élevage de porcs B (transport, abattoir, découpe *) à la Réunion

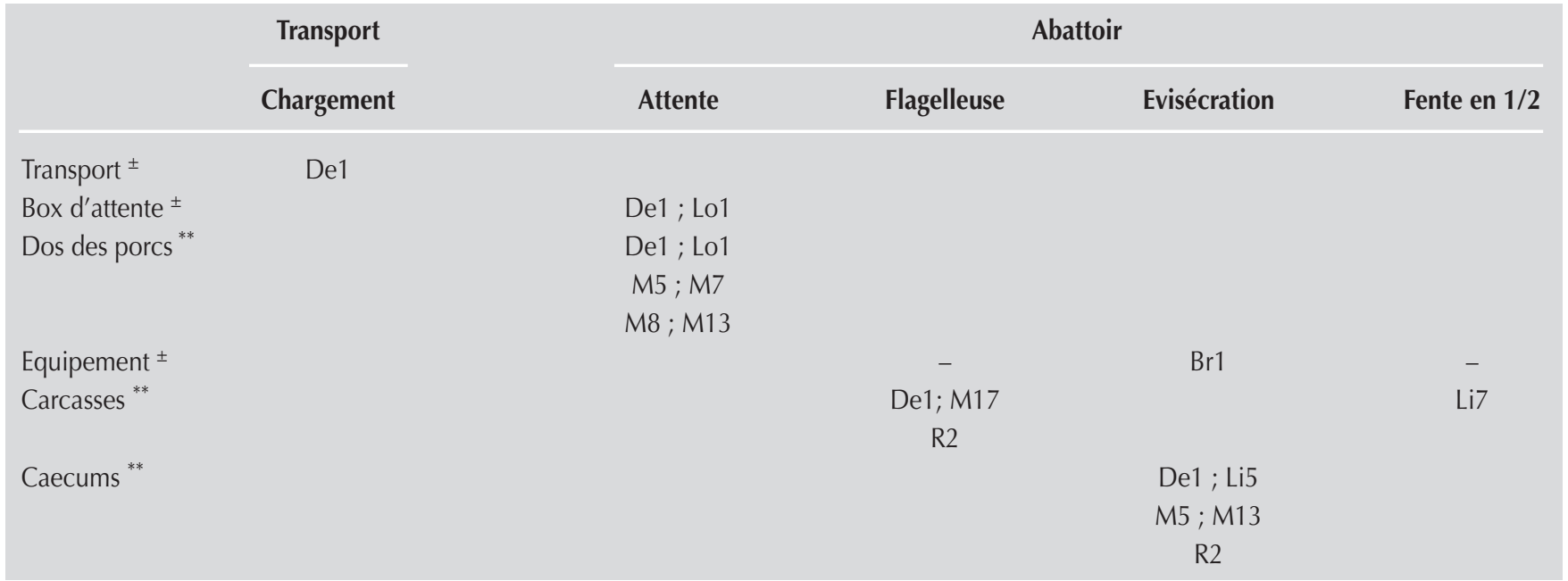

Les différents sérotypes de Salmonella ont été indiqués par des lettres (Br : Bredeney ; De : Derby ; Li : Livingstone ; Lo : London ; M : 4,[5],12:i:- ; R : Rissen) suivies du numéro du pulsotype.

* Tous les prélèvements de l'atelier de découpe (froid choc, $1^{\text {re }}$ et $2^{\mathrm{e}}$ découpes) ont été négatifs.

** Echantillonné après l'étape indiquée dans le tableau

${ }^{ \pm}$Echantillonné après nettoyage et désinfection avant passage du lot

- : Pas de Salmonella 
Ces résultats suggèrent que la contamination environnementale résiduelle est la principale source d'infection des porcs pendant la phase de croissance. Certains pulsotypes isolés des animaux ont été identiques à ceux isolés aux abords des bâtiments d'élevage ou dans d'autres productions animales présentes sur le site. Les pulsotypes identifiés à l'abattoir ont indiqué que la contamination des carcasses provenait soit des souches excrétées par les animaux à l'élevage, soit de contaminations croisées à l'étape de transport et d'attente à l'abattoir. Les carcasses après l'étape de froid-choc et les produits finis étaient tous négatifs.

Cette étude épidémiologique basée sur une analyse moléculaire a permis d'obtenir une photographie de la diffusion de Salmonella tout au long de la filière porcine. La quantification de Salmonella par la méthode de mini-MSRV a montré une variabilité au niveau du taux et de la durée d'excrétion. Les truies ne sont pas la principale source d'infection des porcs au niveau de l'élevage. Un environnement insuffisamment nettoyé et désinfecté sur le site d'élevage, pendant le transport ou pendant l'attente à l'abattoir peut contribuer significativement à l'infection des porcs.

Cette étude, réalisée pour la première fois dans la filière porcine à la Réunion, a permis de cibler les principales voies de contamination et ainsi d'identifier les mesures à mettre en place tout au long de la filière porcine pour limiter au maximum les contaminations par Salmonella.

\section{BIBLIOGRAPHIE}

1. CARDINALE E., MAEDER S., DEBOUIN M., PORPHYRE V., 2010. Salmonella infections in fattening pigs in La Reunion Island: herd prevalence and risk factors for infection. Prev. Vet. Med., 96: 281-285.

2. EUROPEAN FOOD SAFETY AUTHORITY AND EUROPEAN CENTRE FOR DISEASE PREVENTION AND CONTROL, 2014. The European Union summary report on trends and sources of zoonoses, zoonotic agents and food-borne outbreaks in 2012. EFSA J., 12: 312.

3. FRAVALO P., HASCOET Y., FELLIC M., QUEGUINER S., PETTON J., SALVAT G., 2003. Convenient method for rapid and quantitative assessment of Salmonella enterica contamination: the mini-MSRV MPN technique. J. Rapid Methods Autom. Microbiol, 11: 81-88.

4. INTERNATIONAL ORGANIZATION FOR STANDARDIZATION 2002. ISO 6579:2002. Microbiology of food and animal feeding stuffs Horizontal method for the detection of Salmonella spp. London, UK, British Standard Institute.

5. RIBOT E.M., FAIR M.A., GAUTOM R., CAMERON D.N., HUNTER S.B., SWAMINATHAN B., BARRETT T.J., 2006. Standardization of pulsedfield gel electrophoresis protocols for the subtyping of Escherichia coli O157:H7, Salmonella, and Shigella for PulseNet. Foodborne Pathog. Dis., 3: $59-67$.

Accepted 23 January 2015; Online publication June 2015 